\title{
The Kustaanheimo-Stiefel transformation in geometric algebra
}

\author{
T Bartsch \\ Institut für Theoretische Physik 1, Universität Stuttgart, 70550 Stuttgart, \\ Germany \\ E-mail: bartsch@theo1.physik.uni-stuttgart.de
}

\begin{abstract}
The Kustaanheimo-Stiefel (KS) transformation maps the non-linear and singular equations of motion of the three-dimensional Kepler problem to the linear and regular equations of a four-dimensional harmonic oscillator. It is used extensively in studies of the perturbed Kepler problem in celestial mechanics and atomic physics. In contrast to the conventional matrix-based approach, the formulation of the KS transformation in the language of geometric Clifford algebra offers the advantages of a clearer geometrical interpretation and greater computational simplicity. It is demonstrated that the geometric algebra formalism can readily be used to derive a Lagrangian and Hamiltonian description of the KS dynamics in arbitrary static electromagnetic fields. For orbits starting at the Coulomb centre, initial conditions are derived and a framework is set up that allows a discussion of the stability of these orbits.

PACS numbers: 45.20.Jj,31.25.Gy,45.50.Pk
\end{abstract}

\section{Introduction}

The Kepler problem belongs to the simplest systems of classical mechanics. A detailed understanding of its properties is of equally fundamental importance to celestial mechanics and to atomic physics. In both areas it is of interest to describe, either by means of analytic approximations or numerical computation, the impact of additional non-Coulombic forces on the dynamics. In its original form, the equation of motion

$$
\frac{\mathrm{d}^{2} \boldsymbol{x}}{\mathrm{d} t^{2}}=-\frac{\boldsymbol{x}}{|\boldsymbol{x}|^{3}}
$$

is not well suited to this purpose because it is highly non-linear and exhibits a singularity at the Coulomb centre where the force diverges. For numerical studies of the dynamics it is mandatory to find a representation of the equations of motion which avoids this singularity.

For the one-dimensional Kepler motion, it was already found by Euler [1] that the introduction of a square-root coordinate $u=\sqrt{x}$ and a fictitious time $\tau$ defined by $\mathrm{d} t=x \mathrm{~d} \tau$ reduces the Kepler equation of motion (1) to the equation of motion of a one-dimensional harmonic oscillator

$$
\frac{\mathrm{d}^{2} u}{\mathrm{~d} s^{2}}+2 E u=0
$$


where $E$ is the energy of the Kepler motion. Equation (2) is not only void of singularities, it is also linear and thus forms a much more convenient basis for analytic calculations.

Generalizing this approach, Levi-Cività 22 regularized the two-dimensional Kepler problem by combining the two spatial coordinates into a complex number $x=x_{1}+\mathrm{i} x_{2}$ and introducing a complex square-root coordinate $u=\sqrt{x}$, which together with the fictitious-time transformation $\mathrm{d} t=|x| \mathrm{d} \tau$ reduces the Kepler problem to a two-dimensional harmonic oscillator.

Attempts to extend this regularization scheme to the three-dimensional Kepler problem failed, until in 1964 Kustaanheimo and Stiefel 34] proposed the introduction of four regularizing coordinates instead of three and thereby achieved the reduction of the three-dimensional Kepler problem to a four-dimensional harmonic oscillator. This transformation, which is known as the Kustaanheimo-Stiefel (KS) transformation, is discussed in detail in the monograph by Stiefel and Scheifele [5. Beyond its importance to celectial mechanics, it has proven to be an essential tool for investigating the complicated classical dynamics of the hydrogen atom in crossed electric and magnetic fields [6, 7, 8].

Customarily, the KS transformation is expressed in the language of matrix algebra, which not only necessitates awkward computations of vector and matrix components, but also lacks a transparent geometric interpretation. An alternative formulation in terms of the geometric algebra of Euclidean three-space was introduced by Hestenes [9]. In this formalism, the four KS coordinates are interpreted as the components of a position spinor and are thus given a clear geometric meaning. In addition, the formalism offers computational advantages over the conventional matrixbased approach because it unites the four coordinates into a single spinor.

In this paper I elaborate on the geometric algebra formulation of the KS transformation and work out the details necessary for applications to atomic physics, in particular semiclassical closed-orbit theory [10,11,12,13, 14, 15]. The calculations will amply demonstrate the advantages of the geometric algebra over the matrixbased formalism. The terminology used is adapted to applications in atomic physics. In particular, I refer to the attractive centre as the nucleus and discuss the motion of an electron of unit mass and negative unit charge under the influence of the nucleus and arbitrary static external electromagnetic fields. Nevertheless, the results are of much broader range. They apply equally to celestial mechanics or any other field of physics governed by the equations of motion of a perturbed Kepler problem.

In section 2. the geometric algebra formulation of the $\mathrm{KS}$ transformation is introduced and its relation to the matrix-based approch is established. The spinor equation of motion given by Hestenes [9] is derived. In section 3] a Lagrangian and Hamiltonian formulation of the KS spinor dynamics is derived. It cannot be obtained by a simple change of variables because the KS transformation introduces a non-physical fourth degree of freedom and a pseudotime parameter. Nevertheless, it is demonstrated that the geometric algebra formulation readily lends itself to an incorporation into the Lagrangian and Hamiltonian formulations of dynamics. At the same time, the well-known KS Hamiltonian [12, 13, 6], which is restricted to homogeneous external electromagnetic fields, is generalized to arbitrary static fields. Section 4 presents the explicit solution of the spinor equation of motion in a pure Coulomb field and gives the constants of motion in terms of the KS spinor. Section 5 discusses the KS description of orbits starting at the Coulomb centre and returning to it. These orbits, referred to as "closed orbits", are of particular importance because 
they play a central role in the semiclassical interpretation of atomic photo-absorption spectra 10, 11, 12, 13. They require a special treatment because, although the spinor equation of motion is regular at the nucleus, the KS transform is singular there. I will derive initial conditions for orbits starting at the nucleus and present a basis of the spinor space that is suitable for the investigation of the stability of closed orbits. A brief introduction into the properties of geometric algebra needed here is given in Appendix A where the notation used in what follows is also explained. A more detailed exposition of the formalism can be found in 9, 16, 17, 18.

\section{The spinor equation of motion}

The KS-transformation in three dimensions can be found by representing an arbitrary position in space not by its position vector $\boldsymbol{x}$, but by a position spinor, i.e. the rotation-dilatation operator transforming a fixed reference vector into the position vector $\boldsymbol{x}$. As explained in Appendix A a rotation-dilatation of the reference vector $\sigma_{3}$ is represented in the geometric algebra by an even multivector $U$ according to

$$
\boldsymbol{x}=\frac{1}{2} U \boldsymbol{\sigma}_{3} U^{\dagger} .
$$

The factor $1 / 2$ was introduced here to stay in touch with earlier applications of the KS-transformation to atomic dynamics 12,13, although the present formulation of the theory would suggest dropping it. It implies the normalization

$$
U^{\dagger} U=U U^{\dagger}=2 r=2|\boldsymbol{x}| .
$$

Up to normalization, the ansatz (3) reproduces the square-root coordinates introduced by Euler and Levi-Cività, respectively, if it is applied to spaces of one or two dimensions.

Given a position vector $\boldsymbol{x}$, the choice of the spinor $U$ is not unique. More precisely, the gauge transformation

$$
U \mapsto U \mathrm{e}^{-I_{3} \alpha / 2}
$$

with arbitrary real $\alpha$ does not alter $\boldsymbol{x}$, because the additional exponential factor describes a rotation of the reference vector $\sigma_{3}$ around itself. This consideration immediately clarifies why a position spinor representation in three dimensions must introduce a fourth degree of freedom. In lower dimensions, a rotation does not leave any vector invariant, so that the spinor transformation does not possess a gauge degree of freedom. It is also clear from (515) that all fibres of the KS transformation except $U=0$ are circles in spinor space.

The inverse KS transformation can be found from equation A.13 by adapting the normalization to (4). The position spinors corresponding to a vector $\boldsymbol{x}$ are given by

$$
U=\frac{r+\boldsymbol{x} \boldsymbol{\sigma}_{3}}{\sqrt{r+z}} \mathrm{e}^{-I_{3} \alpha / 2}
$$

with arbitrary real $\alpha$.

In components, the spinor $U$ can be represented as $U=u_{0}+I \boldsymbol{u}$ with $\boldsymbol{u}=$ $\sum_{k=1}^{3} u_{k} \boldsymbol{\sigma}_{k}$. The transformation (3) then decomposes into

$$
\begin{aligned}
& x=u_{1} u_{3}-u_{0} u_{2}, \\
& y=u_{1} u_{0}+u_{2} u_{3}, \\
& z=\frac{1}{2}\left(u_{0}^{2}-u_{1}^{2}-u_{2}^{2}+u_{3}^{2}\right) .
\end{aligned}
$$


Up to renumbering the components, this agrees with the conventions of [12,13].

To obtain an equation of motion for $U$, time derivatives of $U$ must be calculated. Differentiating (3) leads to

$$
\dot{\boldsymbol{x}}=\frac{1}{2} \dot{U} \boldsymbol{\sigma}_{3} U^{\dagger}+\frac{1}{2} U \boldsymbol{\sigma}_{3} \dot{U}^{\dagger}=\left\langle\dot{U} \boldsymbol{\sigma}_{3} U^{\dagger}\right\rangle_{1} .
$$

Equation (8) obviously cannot be solved for $\dot{U}$ because the time derivative of the gauge parameter $\alpha$ in (5) cannot be determined from the dynamics of the position vector. To arrive at an equation of motion for $U$, I must therefore impose a constraint on $\alpha$. This can be done in a convenient and geometrically appealing fashion by requiring

$$
\left\langle\dot{U} \boldsymbol{\sigma}_{3} U^{\dagger}\right\rangle_{3}=0
$$

which means that $\dot{U}$ is chosen such as not to contain a component of rotation around the instantaneous position vector $\boldsymbol{x}$. Under this constraint, (8) yields

$$
\dot{\boldsymbol{x}}=\dot{U} \boldsymbol{\sigma}_{3} U^{\dagger}
$$

and

$$
\dot{U}=\dot{\boldsymbol{x}} U^{\dagger}{ }^{-1} \boldsymbol{\sigma}_{3}=\dot{\boldsymbol{x}} \frac{U}{2 r} \boldsymbol{\sigma}_{3} .
$$

As in the one- and two-dimensional cases, the regularization of the threedimensional Kepler motion requires the introduction of a fictitious-time parameter $\tau$. It is defined by

$$
\mathrm{d} t=2 r \mathrm{~d} \tau
$$

Derivatives with respect to $\tau$ will be denoted with a prime. Equation (11) then yields

$$
U^{\prime}=2 r \dot{U}=\dot{\boldsymbol{x}} U \boldsymbol{\sigma}_{3} .
$$

For the second derivative of $U$, I obtain

$$
\begin{aligned}
U^{\prime \prime} & =\left(\frac{d}{d \tau} \dot{\boldsymbol{x}}\right) U \boldsymbol{\sigma}_{3}+\dot{\boldsymbol{x}} U^{\prime} \boldsymbol{\sigma}_{3} \\
& =2 r \ddot{\boldsymbol{x}} U \boldsymbol{\sigma}_{3} \frac{U^{\dagger}}{2 r} U+\dot{\boldsymbol{x}}^{2} U \\
& =2\left(\ddot{\boldsymbol{x}} \boldsymbol{x}+\frac{1}{2} \dot{\boldsymbol{x}}^{2}\right) U .
\end{aligned}
$$

Together with Newton's equation of motion

$$
\ddot{\boldsymbol{x}}=-\frac{\boldsymbol{x}}{r^{3}}+\boldsymbol{f}
$$

with an arbitrary non-Coulombic force $f$, equation (14) yields the spinor equation of motion in the form first given by Hestenes 9 :

$$
U^{\prime \prime}=2\left(E_{\mathrm{K}}+\boldsymbol{f} \boldsymbol{x}\right) U,
$$

where the Kepler energy

$$
E_{\mathrm{K}}=\frac{1}{2} \dot{\boldsymbol{x}}^{2}-\frac{1}{r}
$$

denotes the sum of the kinetic and Coulombic potential energies.

In the special case of pure Kepler motion, i.e. $\boldsymbol{f}=0$, the Kepler energy $E_{\mathrm{K}}$ is equal to the total energy $E$ and is conserved. In this case, (16) reduces to the linear equation of motion

$$
U^{\prime \prime}=2 E U .
$$


If $E<0$, this is the equation of motion of a four-dimensional isotropic harmonic oscillator with frequency $\omega=\sqrt{-2 E}$ with respect to $\tau$.

If additional forces $\boldsymbol{f}$ are present, the Kepler energy is not conserved in general, so that the work done by the external forces must be taken into account 4]. This can easily be achieved if the external forces are generated by static electromagnetic fields, because the work done by a magnetic field $\boldsymbol{B}$ is zero, whereas an electric field $\boldsymbol{F}=-\nabla V$ can be derived from a potential $V(\boldsymbol{x})$. In this case, the total energy $E=E_{\mathrm{K}}-V$ is conserved, so that the equation of motion reads

$$
U^{\prime \prime}=2(E+V(\boldsymbol{x})+\boldsymbol{f} \boldsymbol{x}) U
$$

with $\boldsymbol{f}=-\boldsymbol{F}-\dot{\boldsymbol{x}} \times \boldsymbol{B}$.

It finally remains to verify that the equation of motion (16) is consistent with the constraint (9). To prove this, first note that

$$
\xi=\left\langle U^{\prime} \boldsymbol{\sigma}_{3} U^{\dagger}\right\rangle_{3}
$$

is a constant of motion for any external forces $f$ [4, because by (16)

$$
\begin{aligned}
\frac{d \xi}{d \tau} & =\left\langle U^{\prime \prime} \boldsymbol{\sigma}_{3} U^{\dagger}\right\rangle_{3}+\underbrace{\left\langle U^{\prime} \boldsymbol{\sigma}_{3} U^{\prime \dagger}\right\rangle_{3}}_{=0 \text { by }} \\
& =\left\langle\left(E_{\mathrm{K}}+\boldsymbol{f} \boldsymbol{x}\right) \boldsymbol{x}\right\rangle_{3} \\
& =\left\langle E_{\mathrm{K}} \boldsymbol{x}+r^{2} \boldsymbol{f}\right\rangle_{3} \\
& =0 .
\end{aligned}
$$

Therefore, if the initial conditions are chosen so that $\xi=0$ at $\tau=0$, equation (21) guarantees $\left\langle\dot{U} \boldsymbol{\sigma}_{3} U^{\dagger}\right\rangle_{3}=2 r \xi=0$ at all times.

\section{Canonical formalism}

In classical investigations of atoms in external fields, the Hamiltonian nature of the dynamics plays a central role. It is therefore essential to show how the spinor equation of motion found in the previous section can be derived in the context of a Lagrangian or Hamiltonian formalism. In the matrix theory of the KS transformation, a Hamiltonian formulation is well known and widely applied in the literature 12,13. Due to the introduction of an additional degree of freedom and a fictitious-time parameter, it cannot be found by a straightforward change of variables. In this section it will be shown that the application of geometric algebra allows an easy and general derivation of the Hamiltonian. At the same time, the Hamiltonian formalism will be generalized to arbitrary inhomogeneous static external fields.

\subsection{Fictitious-time transformations}

Elementary expositions of Lagrangian and Hamiltonian dynamics usually treat the time $t$ as the externally prescribed independent variable fundamentally different from the spatial coordinates, velocities, and momenta. The formalisms are then shown to be invariant under point transformations or canonical transformations, respectively, which may be time-dependent, but may not transform the time variable. However, both the Lagrangian and the Hamiltonian formalisms can be reformulated in such a way that it is possible to introduce an arbitrary orbital parameter $\tau$ and to treat the physical time $t$ as an additional coordinate on the same footing as the spatial 
coordinates. This formalism is discussed in its full generality by Dirac [19, 20]. For the special case of autonomous Lagrangian dynamics and the simple form of the fictitious-time transformation used above, the full flexibility of Dirac's homogeneous formalism is not needed. Instead, the modifications needed to achieve the fictitioustime transformation can be derived in a straightforward manner from Hamilton's variational principle.

The Lagrangian equations of motion can be derived from the action functional

$$
S=\int_{t_{1}}^{t_{2}} \mathrm{~d} t L(q(t), \dot{q}(t))
$$

by requiring that for the classical paths the variation of $S$ with respect to the path $q(t)$ vanishes if the variation is performed with the initial and final times $t_{1}$ and $t_{2}$ and the coordinates $q\left(t_{1}\right)$ and $q\left(t_{2}\right)$ kept fixed. If a fictitious-time parameter $\tau$ is introduced by the prescription

$$
\mathrm{d} t=f(q, \dot{q}) \mathrm{d} \tau
$$

with an arbitrary function $f$, it is tempting to rewrite the action functional as

$$
S=\int_{\tau_{1}}^{\tau_{2}} \mathrm{~d} \tau f(q, \dot{q}) L(q, \dot{q})
$$

and regard

$$
\tilde{L}=f(q, \dot{q}) L(q, \dot{q})=\frac{\mathrm{d} t}{\mathrm{~d} \tau} L
$$

as the Lagrangian describing the dynamics with respect to $\tau$. However, this simple procedure is incorrect in general, because to derive the Lagrangian equations with respect to $\tilde{L}$ from (24), the variation of $S$ has to be performed with the initial and final fictitious times $\tau_{1}$ and $\tau_{2}$ kept fixed, and due to (23) a variation of the path will alter the relation between $t$ and $\tau$, so that the initial and final physical times $t_{1}$ and $t_{2}$ will vary.

To establish the true relation between (22) and (24), I calculate the variation of (24) taking the variation of $t$ into account, i.e. $q$ and $t$ are varied according to

$$
\begin{aligned}
& q(\tau) \mapsto q(\tau)+\delta q(\tau), \\
& t(\tau) \mapsto t(\tau)+\delta t(\tau),
\end{aligned}
$$

subject to the boundary conditions

$$
\delta q\left(\tau_{1}\right)=\delta q\left(\tau_{2}\right)=0
$$

and with $\tau_{1}$ and $\tau_{2}$ kept fixed. Under this variation,

$$
\begin{aligned}
\frac{\mathrm{d} \tau}{\mathrm{d} t} \mapsto \frac{\mathrm{d} \tau}{\mathrm{d}(t+\delta t)} & =\frac{1}{\frac{\mathrm{d} t}{\mathrm{~d} \tau}+\frac{\mathrm{d} \delta t}{\mathrm{~d} \tau}} \\
& =\frac{\mathrm{d} \tau}{\mathrm{d} t}\left(1-\frac{\mathrm{d} \tau}{\mathrm{d} t} \frac{\mathrm{d} \delta t}{\mathrm{~d} \tau}\right),
\end{aligned}
$$

so that

$$
\delta \frac{\mathrm{d} \tau}{\mathrm{d} t}=-\left(\frac{\mathrm{d} \tau}{\mathrm{d} t}\right)^{2} \frac{\mathrm{d} \delta t}{\mathrm{~d} \tau}
$$


and hence

$$
\begin{aligned}
\delta \dot{q} & =\delta\left(\frac{\mathrm{d} q}{\mathrm{~d} \tau} \frac{\mathrm{d} \tau}{\mathrm{d} t}\right) \\
& =\delta\left(\frac{\mathrm{d} q}{\mathrm{~d} \tau}\right) \cdot \frac{\mathrm{d} \tau}{\mathrm{d} t}+\frac{\mathrm{d} q}{\mathrm{~d} \tau} \cdot \delta\left(\frac{\mathrm{d} \tau}{\mathrm{d} t}\right) \\
& =\frac{\mathrm{d} \delta q}{\mathrm{~d} t}-\dot{q} \frac{\mathrm{d} \tau}{\mathrm{d} t} \frac{\mathrm{d} \delta t}{\mathrm{~d} \tau} .
\end{aligned}
$$

The variation of (24) then reads

$$
\begin{aligned}
\delta S & =\int \mathrm{d} \tau\left[\delta\left(\frac{\mathrm{d} t}{\mathrm{~d} \tau}\right) L+\frac{\mathrm{d} t}{\mathrm{~d} \tau}\left(\frac{\partial L}{\partial q} \delta q+\frac{\partial L}{\partial \dot{q}} \delta \dot{q}\right)\right] \\
& =\int \mathrm{d} \tau \frac{\mathrm{d} \delta t}{\mathrm{~d} \tau}\left(L-\dot{q} \frac{\partial L}{\partial \dot{q}}\right)+\int \mathrm{d} t\left(\frac{\partial L}{\partial q} \delta q+\frac{\partial L}{\partial \dot{q}} \frac{\mathrm{d} \delta q}{\mathrm{~d} t}\right) \\
& =-\int \mathrm{d} \tau \frac{\mathrm{d} \delta t}{\mathrm{~d} \tau} H+\int \mathrm{d} t\left(\frac{\partial L}{\partial q}-\frac{\mathrm{d}}{\mathrm{d} t} \frac{\partial L}{\partial \dot{q}}\right) \delta q,
\end{aligned}
$$

where the customary partial integration was performed, the boundary conditions (27) were used and the Hamiltonian

$$
H=\dot{q} \frac{\partial L}{\partial \dot{q}}-L
$$

was introduced.

If only the second integral in the last line of (31) were present, it would yield the correct equations of motion. Thus, the action functionals (22) and (24) are equivalent if the Hamiltonian $H$ vanishes. For autonomous systems, $H$ is a constant of motion equal to the energy $E$. If the Lagrangian $L$ is replaced with $L+E$, with $E$ regarded as a constant, the equations of motion derived from $L$ are unchanged, but the Hamiltonian (32) changes to $H-E=0$. Thus, the dynamics of trajectories with energy $E$ with respect to the fictitious-time parameter $\tau$ is described by the Lagrangian

$$
\mathcal{L}=\frac{\mathrm{d} t}{\mathrm{~d} \tau}(L+E)=f(q, \dot{q})(L+E) .
$$

This Lagrangian has to be written as a function of the coordinates $q$ and the fictitioustime velocities $q^{\prime}$. If the function $f$ is independent of the velocities, the canonical momenta are invariant under the fictitious-time transformation, because $q^{\prime}=f(q) \dot{q}$ and

$$
\frac{\partial \mathcal{L}}{\partial q^{\prime}}=f(q) \frac{\mathrm{d} \dot{q}}{\mathrm{~d} q^{\prime}} \frac{\partial L}{\partial \dot{q}}=\frac{\partial L}{\partial \dot{q}} .
$$

From the time-transformed Lagrangian (33), the transformed Hamiltonian

$$
\mathcal{H}=q^{\prime} \frac{\partial \mathcal{L}}{\partial q^{\prime}}-\mathcal{L}=f(q, \dot{q})(H-E)
$$

is obtained by the usual Legendre transformation. It must be written as a function of the coordinates and momenta. In some cases the passage from the Lagrangian to the Hamiltonian description of the dynamics is impossible because the relation $p=\partial \mathcal{L}\left(q, q^{\prime}\right) / \partial q^{\prime}$ cannot be solved for $q^{\prime}$. In these cases, the Hamiltonian (35) can be shown to describe the fictitious-time dynamics by a discussion of the modified Hamilton's principle analogous to the derivation of the Lagrangian $\mathcal{L}$ above. 


\subsection{Lagrangian description}

The dynamics of an atomic electron under the combined influences of the nuclear Coulomb potential, an additional scalar potential $V(\boldsymbol{x})$ and a magnetic field represented by a vector potential $\boldsymbol{A}(\boldsymbol{x})$ is described by the Lagrangian

$$
L=\frac{\dot{\boldsymbol{x}}^{2}}{2}+\frac{1}{r}+V(\boldsymbol{x})-\boldsymbol{A} \cdot \dot{\boldsymbol{x}} .
$$

This Lagrangian must be transformed to a Lagrangian $\mathcal{L}$ describing the fictitious-time dynamics of the position spinor $U$. With $f(q)=2 r=U^{\dagger} U$ by (12), the fictitious-time Lagrangian (33) reads

$\mathcal{L}=\frac{1}{4} U^{\prime \dagger} U^{\prime}+\frac{1}{8 r}\left\langle\left(U^{\prime} \boldsymbol{\sigma}_{3} U^{\dagger}\right)^{2}\right\rangle+E U^{\dagger} U+U^{\dagger} U V(\boldsymbol{x})-\left\langle\boldsymbol{A}(\boldsymbol{x}) U^{\prime} \boldsymbol{\sigma}_{3} U^{\dagger}\right\rangle+2$

with $\boldsymbol{x}=\frac{1}{2} U \boldsymbol{\sigma}_{3} U^{\dagger}$. If the constraint (9) is used, $\mathcal{L}$ simplifies to

$\mathcal{L}=\frac{1}{2} U^{\prime \dagger} U^{\prime}+E U^{\dagger} U+U^{\dagger} U V(\boldsymbol{x})-\left\langle\boldsymbol{A}(\boldsymbol{x}) U^{\prime} \boldsymbol{\sigma}_{3} U^{\dagger}\right\rangle+2$

Both forms of the Lagrangian yield the same "on-shell" dynamics for trajectories satisfying (91). Note that only the kinetic term is influenced by the constraint, whereas potential and vector potential terms are not.

The momentum conjugate to $U$ is given by

$$
P=\partial_{U^{\prime}} \mathcal{L}=\frac{1}{2 r} \boldsymbol{\sigma}_{3} U^{\dagger}\left\langle U^{\prime} \boldsymbol{\sigma}_{3} U^{\dagger}\right\rangle_{1}-\boldsymbol{\sigma}_{3} U^{\dagger} \boldsymbol{A},
$$

which simplifies to

$$
P=U^{\prime \dagger}-\sigma_{3} U^{\dagger} \boldsymbol{A}
$$

if (9) is applied.

As the spinor equation of motion (16) is valid under the constraint (9) only, the Lagrangian $\mathcal{L}$ provides a suitable description of the dynamics if it reproduces (16) for trajectories satisfying (9). The simplified Lagrangian (38) can therefore be used. When equations of motion are derived from (38), the constraint (9) must be taken into account by a Lagrangian multiplier. I will now show, however, that the unconstrained equation of motion [17.

$$
\frac{d}{d \tau} \partial_{U^{\prime}} \mathcal{L}-\partial_{U} \mathcal{L}=0
$$

derived from (38) reproduces (16) without the constraint being explicitly dealt with, i.e. the Lagrangian multiplier to be introduced turns out to vanish identically. I therefore ignore it from the outset.

For the case of vanishing external potentials, (41) can easily be seen to yield

$$
U^{\prime \prime \dagger}-2 E U^{\dagger}=0,
$$

which is the reversion of (18). For the terms containing the potentials, the calculation of (41) is still straightforward, but requires a more intimate familiarity with the properties of the multivector derivative. I will therefore present the calculation in detail.

The contribution of the scalar potential term

$$
\mathcal{V}=U^{\dagger} U V(\boldsymbol{x}(U))
$$


with $\boldsymbol{x}(U)=\frac{1}{2} U \boldsymbol{\sigma}_{3} U^{\dagger}$ reads, by A.22,

$$
\partial_{U} \mathcal{V}=2 U^{\dagger} V(\boldsymbol{x})+U^{\dagger} U \partial_{U} V(\boldsymbol{x}(U)) .
$$

The chain rule (A.24) then yields for any even multivector $M$

$$
M * \partial_{U} V(\boldsymbol{x}(U))=\left(M * \partial_{U} \boldsymbol{x}(U)\right) * \partial_{\boldsymbol{x}} V
$$

with

$$
\begin{aligned}
M * \partial_{U} \boldsymbol{x}(U) & =\left(M * \partial_{U}\right) \frac{1}{2} U \boldsymbol{\sigma}_{3} U^{\dagger} \\
& =\frac{1}{2} M \boldsymbol{\sigma}_{3} U^{\dagger}+\frac{1}{2} U \boldsymbol{\sigma}_{3} M^{\dagger} \\
& =\left\langle M \boldsymbol{\sigma}_{3} U^{\dagger}\right\rangle_{1} .
\end{aligned}
$$

In the absence of a magnetic field the external force is $\boldsymbol{f}=\partial_{\boldsymbol{x}} V$, so that

$$
M * \partial_{U} V(\boldsymbol{x}(U))=\left\langle\left\langle M \boldsymbol{\sigma}_{3} U^{\dagger}\right\rangle_{1} \boldsymbol{f}\right\rangle=\left\langle M \boldsymbol{\sigma}_{3} U^{\dagger} \boldsymbol{f}\right\rangle .
$$

Thus,

$$
\partial_{U} V=\partial_{M}\left(M * \partial_{U} V\right)=\boldsymbol{\sigma}_{3} U^{\dagger} \boldsymbol{f},
$$

and finally

$$
\partial_{U} \mathcal{V}=2 U^{\dagger} V(\boldsymbol{x})+2 U^{\dagger} \boldsymbol{x} \boldsymbol{f} .
$$

This is the reversion of the scalar-potential terms in (16). Therefore, (49) together with (42) indeed yields the correct equation of motion.

To evaluate the contribution of the vector potential term

$$
\mathcal{A}=\left\langle\boldsymbol{A}(\boldsymbol{x}) U^{\prime} \boldsymbol{\sigma}_{3} U^{\dagger}\right\rangle
$$

to (41), first note that

$$
\begin{aligned}
\frac{d}{d \tau} \partial_{U^{\prime}} \mathcal{A} & =\frac{d}{d \tau}\left(\boldsymbol{\sigma}_{3} U^{\dagger} \boldsymbol{A}(\boldsymbol{x})\right) \\
& =\boldsymbol{\sigma}_{3} U^{\prime \dagger} \boldsymbol{A}(\boldsymbol{x})+\boldsymbol{\sigma}_{3} U^{\dagger}\left(\boldsymbol{x}^{\prime} \cdot \partial_{\boldsymbol{x}}\right) \boldsymbol{A}(\boldsymbol{x}) .
\end{aligned}
$$

By Leibniz' rule and (8),

$$
\begin{aligned}
\partial_{U} \mathcal{A} & =\stackrel{*}{\partial} U\left\langle\boldsymbol{A} U^{\prime} \boldsymbol{\sigma}_{3} U^{\dagger}\right\rangle+\stackrel{*}{\partial} U\left\langle\stackrel{*}{\boldsymbol{A}} U^{\prime} \boldsymbol{\sigma}_{3} U^{\dagger}\right\rangle \\
& =\boldsymbol{\sigma}_{3} U^{\prime \dagger} \boldsymbol{A}+\partial_{U}\left\langle\boldsymbol{A}(\boldsymbol{x}(U)) \boldsymbol{x}^{\prime}\right\rangle .
\end{aligned}
$$

The second term on the right-hand side can be evaluated by first calculating directional derivatives. For an arbitrary even $M$, (46) and the chain rule (A.24) yield

$$
\begin{aligned}
\left(M * \partial_{U}\right)\left\langle\boldsymbol{A}(\boldsymbol{x}(U)) \boldsymbol{x}^{\prime}\right\rangle & =\left(M * \partial_{U} \boldsymbol{A}\right) * \partial_{\boldsymbol{A}}\left\langle\boldsymbol{A} \boldsymbol{x}^{\prime}\right\rangle \\
& =\left\langle\left(M * \partial_{U} \boldsymbol{A}(\boldsymbol{x}(U))\right) \boldsymbol{x}^{\prime}\right\rangle \\
& =\left\langle\left(M * \partial_{U} \boldsymbol{x}\right) * \partial_{\boldsymbol{x}} \boldsymbol{A} \boldsymbol{x}^{\prime}\right\rangle \\
& =\left\langle\left\langle\left\langle M \boldsymbol{\sigma}_{3} U^{\dagger}\right\rangle_{1} \partial_{\boldsymbol{x}}\right\rangle \boldsymbol{A} \boldsymbol{x}^{\prime}\right\rangle \\
& =\left\langle M \boldsymbol{\sigma}_{3} U^{\dagger} \partial_{\boldsymbol{x}}\right\rangle\left\langle\boldsymbol{A} \boldsymbol{x}^{\prime}\right\rangle,
\end{aligned}
$$

so that

$$
\begin{aligned}
\partial_{U}\left\langle\boldsymbol{A}(\boldsymbol{x}(U)) \boldsymbol{x}^{\prime}\right\rangle & =\partial_{M}\left(M * \partial_{U}\right)\left\langle\boldsymbol{A}(\boldsymbol{x}(U)) \boldsymbol{x}^{\prime}\right\rangle \\
& =\boldsymbol{\sigma}_{3} U^{\dagger} \partial_{\boldsymbol{x}}\left(\boldsymbol{A} \cdot \boldsymbol{x}^{\prime}\right) .
\end{aligned}
$$


Equations (51), (52) and (54) combine to

$$
\begin{aligned}
\frac{d}{d \tau} \partial_{U^{\prime}} \mathcal{A}-\partial_{U} \mathcal{A} & =\boldsymbol{\sigma}_{3} U^{\dagger}\left(\left(\boldsymbol{x}^{\prime} \cdot \partial_{\boldsymbol{x}}\right) \boldsymbol{A}-\partial_{\boldsymbol{x}}\left(\boldsymbol{A} \cdot \boldsymbol{x}^{\prime}\right)\right) \\
& =-\boldsymbol{\sigma}_{3} U^{\dagger}\left(\boldsymbol{x}^{\prime} \times\left(\partial_{\boldsymbol{x}} \times \boldsymbol{A}\right)\right) \\
& =-2 U^{\dagger} \boldsymbol{x}(\dot{\boldsymbol{x}} \times \boldsymbol{B})
\end{aligned}
$$

which is the reversion of the magnetic-field contribution to (16).

Note that the derivation given here is valid for arbitrary external potentials $V$ and $\boldsymbol{A}$, whereas conventional treatments restrict themselves to the special case of homogeneous external fields. It can obviously be further generalized to include conservative forces of non-electromagnetic origin. Also note that the geometric algebra formalism allows one to do the calculations in a straightforward manner without having to resort to component decompositions of any of the vectorial or spinorial quantities involved.

\subsection{Hamiltonian description}

The transition from a Lagrangian to a Hamiltonian description of the dynamics leads from the Lagrangian (37) or (38), depending on whether or not the constraint (9) is applied, to the Hamiltonian

$$
\mathcal{H}=\left(U^{\prime} * \partial_{U^{\prime}}\right) \mathcal{L}-\mathcal{L},
$$

in which the velocity $U^{\prime}$ has to be expressed in terms of the momentum $P$. The transformation requires that the relation (39) or (40) between velocity and momentum can be solved for the velocity, which is impossible in the case of (39). Thus, the constraint (9) is not only needed to obtain an unambiguous equation of motion for $U$, but also serves as a condition for a Hamiltonian description of the spinor dynamics to exist. If it is imposed and an inessential constant of 2 is added, the Hamiltonian reads

$$
\mathcal{H}=\frac{1}{2}\left(P^{\dagger}+\boldsymbol{A} U \boldsymbol{\sigma}_{3}\right)\left(P+\boldsymbol{\sigma}_{3} U^{\dagger} \boldsymbol{A}\right)-E U^{\dagger} U-U^{\dagger} U V(\boldsymbol{x})=2 .
$$

Because it is time-independent, the Hamiltonian (57) is a constant of motion. To describe the physical dynamics, its value must be chosen to be 2 , whereas the physical energy $E$ appears as a parameter in $\mathcal{H}$.

The equations of motion derived from (57) read

$$
\begin{aligned}
U^{\prime}= & \partial_{P} \mathcal{H}=P^{\dagger}+\boldsymbol{A} U \boldsymbol{\sigma}_{3}, \\
P^{\prime}= & -\partial_{U} \mathcal{H} \\
= & -2 E U^{\dagger}-2 U^{\dagger} V(\boldsymbol{x})-2 U^{\dagger} \boldsymbol{x} \partial_{\boldsymbol{x}} V \\
& -\boldsymbol{\sigma}_{3}\left(P+\boldsymbol{\sigma}_{3} U^{\dagger} \boldsymbol{A}\right) \boldsymbol{A}-\boldsymbol{\sigma}_{3} U^{\dagger} \stackrel{*}{\partial}_{\boldsymbol{x}}^{*}\left\langle\stackrel{*}{\boldsymbol{A}} \boldsymbol{x}^{\prime}\right\rangle,
\end{aligned}
$$

where

$$
\boldsymbol{x}^{\prime}=U \boldsymbol{\sigma}_{3} U^{\prime \dagger}=U \boldsymbol{\sigma}_{3} P+U^{\dagger} U \boldsymbol{A}
$$

was used. In terms of coordinates and momenta, the constraint (9) reads

$$
\left\langle U \sigma_{3} P\right\rangle_{3}=0 \text {. }
$$

Equation (60) is equivalent to (9) both in the presence and in the absence of a magnetic field. Taken together, (58) and (59) lead back to the equation of motion (16). 
Finally, let me mention an important subtlety regarding the component decomposition of the spinor equation. If, according to (77), $U$ is represented as $U=u_{0}+I \boldsymbol{u}$ with $\boldsymbol{u}=\sum_{k=1}^{3} u_{k} \boldsymbol{\sigma}_{k}$ and $p_{k}$ denotes the momentum component conjugate to $u_{k}$, the spinor momentum is $P=p_{0}-I \boldsymbol{p}$ with $\boldsymbol{p}=\sum_{k=1}^{3} p_{k} \boldsymbol{\sigma}_{k}$. The negative sign is necessary because in the spinor formulation the bivector $I_{k} p_{k}$ is conjugate to $I_{k} u_{k}$. Dropping the bivector factors $I_{k}$ leads to the stated result.

\section{The Kepler problem}

The unperturbed Kepler motion is described by the Hamiltonian

$$
\mathcal{H}=\frac{1}{2} P^{\dagger} P-E U^{\dagger} U=2 .
$$

The equations of motion derived from (61) are

$$
U^{\prime}=P^{\dagger}, \quad P^{\prime}=2 E U^{\dagger}
$$

or, as in (18),

$$
U^{\prime \prime}=2 E U .
$$

If $E<0$, this is the equation of motion of an isotropic four-dimensional harmonic oscillator, whose general solution reads

$$
U=A \cos (\sqrt{-2 E} \tau)+B \sin (\sqrt{-2 E} \tau)
$$

with two constant even multivectors $A$ and $B$ bound, by (91) and (61), to satisfy

$$
\left\langle A \sigma_{3} B^{\dagger}\right\rangle_{3}=0
$$

and

$$
A^{\dagger} A+B^{\dagger} B=-\frac{2}{E} .
$$

In the case of the pure Kepler motion, the angular momentum vector $\boldsymbol{L}$ and the Lenz vector $\boldsymbol{\epsilon}$ are conserved. Together, they uniquely specify an orbit 9 . I will now derive the KS-transformed expressions for these constants of motion. Throughout, the validity of the constraint (9) will be assumed.

The angular momentum vector is given by

$$
\boldsymbol{L}=\boldsymbol{x} \times \dot{\boldsymbol{x}}=-I\langle\boldsymbol{x} \dot{\boldsymbol{x}}\rangle_{2} .
$$

Within the geometric algebra, it is more convenient to introduce the angular momentum bivector

$$
l=I \boldsymbol{L}=\langle\boldsymbol{x} \dot{\boldsymbol{x}}\rangle_{2},
$$

which specifies the orbital plane instead of the direction perpendicular to it. By (3), (8) and (9),

$$
\begin{aligned}
l & =\left\langle\frac{1}{2} U \boldsymbol{\sigma}_{3} U^{\dagger} U \boldsymbol{\sigma}_{3} \dot{U}^{\dagger}\right\rangle_{2} \\
& =\frac{1}{2}\left\langle U U^{\prime \dagger}\right\rangle_{2} \\
& =\frac{1}{2}\langle U P\rangle_{2} .
\end{aligned}
$$


That $l$ is conserved can be verified by a straightforward differentiation. Alternatively, it can be checked that the Poisson bracket vanishes,

$$
\begin{aligned}
\{l, \mathcal{H}\} & =\left(\partial_{P} \mathcal{H}\right) * \partial_{U} l-\left(\partial_{U} \mathcal{H}\right) * \partial_{P} l \\
& =P^{\dagger} * \partial_{U} l+2 E U^{\dagger} * \partial_{P} l \\
& =\frac{1}{2}\left\langle P^{\dagger} P\right\rangle_{2}+E\left\langle U^{\dagger} U\right\rangle_{2} \\
& =0 .
\end{aligned}
$$

Note how the Poisson bracket formalism extends not only to multivector coordinates $U$ and $P$, but also to non-scalar arguments.

The Lenz vector is given by

$$
\begin{aligned}
\boldsymbol{\epsilon} & =l \dot{\boldsymbol{x}}-\frac{\boldsymbol{x}}{r} \\
& =l P^{\dagger} \boldsymbol{\sigma}_{3} U^{-1}-U \boldsymbol{\sigma}_{3} U^{-1} .
\end{aligned}
$$

To calculate the Poisson bracket $\{\boldsymbol{\epsilon}, \mathcal{H}\}$, use $\{l, \mathcal{H}\}=0$ and

$$
P^{\dagger} * \partial_{U} U^{-1}=-U^{-1} P^{\dagger} U^{-1}
$$

to find

$$
\begin{aligned}
\{\boldsymbol{\epsilon}, \mathcal{H}\}= & l\left\{P^{\dagger} \boldsymbol{\sigma}_{3} U^{-1}, \mathcal{H}\right\}-\left\{U \boldsymbol{\sigma}_{3} U^{-1}, \mathcal{H}\right\} \\
= & l\left(P^{\dagger} * \partial_{U}\left(P^{\dagger} \boldsymbol{\sigma}_{3} U^{-1}\right)+2 E U^{\dagger} * \partial_{P}\left(P^{\dagger} \boldsymbol{\sigma}_{3} U^{-1}\right)\right) \\
& -P^{\dagger} * \partial_{U}\left(U \boldsymbol{\sigma}_{3} U^{-1}\right) \\
= & l\left(-P^{\dagger} \boldsymbol{\sigma}_{3} U^{-1} P^{\dagger} U^{-1}+2 E U \boldsymbol{\sigma}_{3} U^{-1}\right) \\
& -P^{\dagger} \boldsymbol{\sigma}_{3} U^{-1}+U \boldsymbol{\sigma}_{3} U^{-1} P^{\dagger} U^{-1} \\
= & {\left[l\left(-P^{\dagger} \boldsymbol{\sigma}_{3} U^{-1} P^{\dagger} \boldsymbol{\sigma}_{3} U^{\dagger}+2 E U U^{\dagger}\right)\right.} \\
& \left.-P^{\dagger} U^{\dagger}+U \boldsymbol{\sigma}_{3} U^{-1} P^{\dagger} \boldsymbol{\sigma}_{3} U^{\dagger}\right] U^{\dagger}{ }^{-1} \boldsymbol{\sigma}_{3} U^{-1}
\end{aligned}
$$

Due to the constraint (60),

$$
P^{\dagger} \boldsymbol{\sigma}_{3} U^{\dagger}=U \boldsymbol{\sigma}_{3} P,
$$

so that equation (72) simplifies to

$$
\begin{aligned}
\{\boldsymbol{\epsilon}, \mathcal{H}\} & =\left[l\left(-P^{\dagger} P+2 E U^{\dagger} U\right)-P^{\dagger} U^{\dagger}+U P\right] U^{\dagger-1} \boldsymbol{\sigma}_{3} U^{-1} \\
& =\left[-2 l \mathcal{H}+2\langle U P\rangle_{2}\right] U^{\dagger^{-1}} \boldsymbol{\sigma}_{3} U^{-1} \\
& =0 .
\end{aligned}
$$

Thus, the Lenz vector $\epsilon$ is actually conserved.

\section{The Kustaanheimo-Stiefel description of closed orbits}

If the initial conditions $\boldsymbol{x}(0)$ and $\dot{\boldsymbol{x}}(0)$ for a trajectory are given, the pertinent initial conditions for the spinors $U$ and $U^{\prime}$ can usually be obtained, up to a choice of gauge, from (6) and (13). This prescription fails for trajectories starting at the origin, where the KS transformation is singular. Although at first sight these trajectories appear rather exceptional, they bear a particular significance to atomic physics: Semiclassical closed-orbit theory [10,11, 15] respresents an atomic photo-absorption spectrum as a sum over closed classical orbits, i.e. orbits that start at the nucleus and return to it. 
Due to that particular importance, closed orbits deserve a detailed discussion. In this section, I will derive initial conditions for a trajectory starting at the Coulomb centre. I will then construct an orthonormal basis of the KS spinor space that is suitable for an investigation of the stability of orbits closed at the centre because it allows to separate the physically distinct directions along and transverse to the orbit from the gauge degree of freedom.

To find initial conditions for orbits starting at the nucleus note that in the vicinity of the nucleus the Coulomb interaction is so strong that it dominates all external forces. The dynamics close to the nucleus is therefore described by the Kepler equation of motion (18) and its solution (63). With the initial condition $U(\tau=0)=0$ implemented, 63) reads

$$
U(\tau)=\frac{U_{0}^{\prime}}{\sqrt{-2 E}} \sin (\sqrt{-2 E} \tau) .
$$

$U_{0}^{\prime}$ is the initial velocity in KS-coordinates. It must be normalized to

$$
U_{0}^{\prime \dagger} U_{0}^{\prime}=4 \text {. }
$$

The choice of gauge for $U_{0}^{\prime}$ is arbitrary.

The position vector corresponding to (75) is

$$
\boldsymbol{x}(\tau)=\frac{1}{2} U_{0}^{\prime} \boldsymbol{\sigma}_{3} U_{0}^{\prime \dagger} \frac{\sin ^{2}(\sqrt{-2 E} \tau)}{-2 E} .
$$

Thus, (75) describes an electron moving out from the nucleus in the direction of the unit vector

$$
s=\frac{1}{4} U_{0}^{\prime} \boldsymbol{\sigma}_{3} U_{0}^{\prime \dagger} .
$$

$U_{0}^{\prime}$ is therefore a spinor rotating the vector $\boldsymbol{\sigma}_{3}$ to the starting direction $\boldsymbol{s}$ and normalized according to (76). In terms of the starting angles $\vartheta$ and $\varphi$ it is given by

$$
U_{0}^{\prime}=2 \mathrm{e}^{-I_{3} \varphi / 2} \mathrm{e}^{-I_{2} \vartheta / 2} \mathrm{e}^{-I_{3} \alpha / 2}
$$

with an arbitrary gauge parameter $\alpha$. The exponentials in (79) describe a sequence of three rotations taking the reference vector $\boldsymbol{\sigma}_{3}$ to the starting direction $\boldsymbol{s}$. The initial momentum reads

$$
P_{0}=U_{0}^{\prime \dagger}=2 \mathrm{e}^{I_{3} \alpha / 2} \mathrm{e}^{I_{2} \vartheta / 2} \mathrm{e}^{I_{3} \varphi / 2} .
$$

Its component decomposition is

$$
\begin{aligned}
& p_{0}=2 \cos \frac{\vartheta}{2} \cos \frac{\varphi+\alpha}{2}, \\
& p_{1}=2 \sin \frac{\vartheta}{2} \sin \frac{\varphi-\alpha}{2}, \\
& p_{2}=-2 \sin \frac{\vartheta}{2} \cos \frac{\varphi-\alpha}{2}, \\
& p_{3}=-2 \cos \frac{\vartheta}{2} \sin \frac{\varphi+\alpha}{2} .
\end{aligned}
$$

To describe the stability of a classical trajectory, a coordinate system with one coordinate along the trajectory and two coordinates perpendicular to it is customarily introduced in the neighbourhood of the trajectory. A linear stability analysis then requires calculating the derivatives of positions and momenta with respect to the 
transverse initial conditions. Most conveniently, derivatives with respect to two orthonormal directions can be used. If these derivatives are to be calculated within the framework of the KS theory, for a given starting direction $s$ and a direction $s_{\omega} \perp s$, a KS spinor $P_{\omega}$ must be found such that a variation of the initial KS momentum $P_{0}$ in the direction of $P_{\omega}$ corresponds to a variation of $s$ in the direction of $s_{\omega}$.

As the initial momentum is given in terms of the starting angles in (80), the derivatives $\partial P_{0} / \partial \vartheta$ and $\partial P_{0} / \partial \varphi$ can be expected to describe variations of the starting direction in the directions of increasing $\vartheta$ and $\varphi$, respectively. To check this and to find the correct normalization of the spinors, I will now construct, for a fixed direction $s$, a basis of the spinor space such that one of the basis spinors describes a variation of initial momentum along the orbit, i.e. in the direction of $s$, two give variations in the directions of two perpendicular unit vectors, and the fourth basis spinor describes the gauge degree of freedom introduced by the KS regularization.

To this end, I consider a family of trajectories parameterized by an arbitrary parameter $\omega$. All trajectories start at the nucleus, the starting direction is given by a family of vectors $s(\omega)$. By (78), the initial KS momenta $P_{0}(\omega)$ then satisfy

$$
s(\omega)=\frac{1}{4} P_{0}^{\dagger}(\omega) \sigma_{3} P_{0}(\omega),
$$

so that in complete analogy with (8) and (11)

$$
\frac{\partial s}{\partial \omega}=\frac{1}{2}\left\langle P_{0}^{\dagger} \sigma_{3} \frac{\partial P_{0}}{\partial \omega}\right\rangle_{1}
$$

and

$$
\frac{\partial P_{0}}{\partial \omega}=\frac{1}{2} \sigma_{3} P_{0} \frac{\partial s}{\partial \omega}=\frac{1}{2} P_{0} s \frac{\partial s}{\partial \omega}
$$

if the gauge condition

$$
\left\langle P_{0}^{\dagger} \boldsymbol{\sigma}_{3} \frac{\partial P_{0}}{\partial \omega}\right\rangle_{3}=0
$$

is imposed. Equation (84) gives the variation in the initial KS momentum pertinent to a given variation in the starting direction. If two different variations are given, the scalar product of the momentum variations is

$$
\left(\frac{\partial P_{0}}{\partial \omega_{1}}\right)^{\dagger} * \frac{\partial P_{0}}{\partial \omega_{2}}=\frac{\partial s}{\partial \omega_{1}} * \frac{\partial s}{\partial \omega_{2}}
$$

so that the variations of KS momentum calculated from (84) are orthonormal if the prescribed variations of the starting direction are.

For a fixed starting direction

$$
s=\mathrm{e}^{-I_{3} \varphi / 2} \mathrm{e}^{-I_{2} \vartheta} \sigma_{3} \mathrm{e}^{I_{3} \varphi / 2}
$$

given by the starting angles $\vartheta$ and $\varphi$, I now introduce the orthogonal vectors

$$
\begin{aligned}
& \boldsymbol{s}_{\vartheta}=\mathrm{e}^{-I_{3} \varphi / 2} \mathrm{e}^{-I_{2} \vartheta} \boldsymbol{\sigma}_{1} \mathrm{e}^{I_{3} \varphi / 2}, \\
& \boldsymbol{s}_{\varphi}=\mathrm{e}^{-I_{3} \varphi / 2} \boldsymbol{\sigma}_{2} \mathrm{e}^{I_{3} \varphi / 2} .
\end{aligned}
$$

These are the unit vectors in the directions of $\partial \boldsymbol{s} / \partial \vartheta$ and $\partial \boldsymbol{s} / \partial \varphi$, respectively. The orthonormal basis $s, s_{\vartheta}$ and $s_{\varphi}$ of the position space gives rise to the three orthonormal KS spinors

$$
\begin{aligned}
& P_{s}=\frac{1}{2} \sigma_{3} P_{0} s=\frac{1}{2} P_{0}, \\
& P_{\vartheta}=\frac{1}{2} \sigma_{3} P_{0} \boldsymbol{s}_{\vartheta}=\frac{I_{2}}{2} \mathrm{e}^{-I_{3} \alpha} P_{0}, \\
& P_{\varphi}=\frac{1}{2} \sigma_{3} P_{0} \boldsymbol{s}_{\varphi}=-\frac{I_{1}}{2} \mathrm{e}^{-I_{3} \alpha} P_{0} .
\end{aligned}
$$


This set is complemented by a fourth orthonormal spinor

$$
P_{\alpha}=\frac{\partial P_{0}}{\partial \alpha}=\frac{1}{2} \sigma_{3} P_{0} I \text {. }
$$

(Note the analogy with (84).) The spinor $P_{\alpha}$ maximally violates the gauge condition (85) in the sense that

$$
P_{0}^{\dagger} \boldsymbol{\sigma}_{3} P_{\alpha}=\left\langle P_{0}^{\dagger} \boldsymbol{\sigma}_{3} P_{\alpha}\right\rangle_{3} .
$$

It therefore gives the direction in spinor space that corresponds to a gauge transformation, whereas $P_{s}$ describes a change of momentum along the orbit (i.e. a change of the energy) and $P_{\vartheta}$ and $P_{\varphi}$ give directions perpendicular to the trajectory. The desired separation of the physically distinct degrees of freedom has thus been achieved. Note that $P_{\vartheta}=\partial P_{0} / \partial \vartheta$ as anticipated, whereas $\partial P_{0} / \partial \varphi=P_{0} I_{3} / 2$ does not satisfy 85. Instead,

$$
\frac{\partial P_{0}}{\partial \varphi}=P_{\alpha} \cos \vartheta+P_{\varphi} \sin \vartheta .
$$

In components, the four basis spinors read

$$
\begin{aligned}
& P_{s}=\quad \cos \frac{\vartheta}{2} \cos \frac{\varphi+\alpha}{2}-I_{1} \sin \frac{\vartheta}{2} \sin \frac{\varphi-\alpha}{2}+I_{2} \sin \frac{\vartheta}{2} \cos \frac{\varphi-\alpha}{2}+I_{3} \cos \frac{\vartheta}{2} \sin \frac{\varphi+\alpha}{2}, \\
& P_{\vartheta}=-\sin \frac{\vartheta}{2} \cos \frac{\varphi+\alpha}{2}-I_{1} \cos \frac{\vartheta}{2} \sin \frac{\varphi-\alpha}{2}+I_{2} \cos \frac{\vartheta}{2} \cos \frac{\varphi-\alpha}{2}-I_{3} \sin \frac{\vartheta}{2} \sin \frac{\varphi+\alpha}{2}, \\
& P_{\varphi}=-\sin \frac{\vartheta}{2} \sin \frac{\varphi+\alpha}{2}-I_{1} \cos \frac{\vartheta}{2} \cos \frac{\varphi-\alpha}{2}-I_{2} \cos \frac{\vartheta}{2} \sin \frac{\varphi-\alpha}{2}+I_{3} \sin \frac{\vartheta}{2} \cos \frac{\varphi+\alpha}{2}, \\
& P_{\alpha}=-\cos \frac{\vartheta}{2} \sin \frac{\varphi+\alpha}{2}+I_{1} \sin \frac{\vartheta}{2} \cos \frac{\varphi-\alpha}{2}+I_{2} \sin \frac{\vartheta}{2} \sin \frac{\varphi-\alpha}{2}+I_{3} \cos \frac{\vartheta}{2} \cos \frac{\varphi+\alpha}{2} .
\end{aligned}
$$

These formulae prescribe a basis of the spinor space uniquely up to the choice of $\alpha$ if $\vartheta \neq 0, \pi$. At the poles, the angle $\varphi$ is undefined. Because in this case (88) gives a pair of orthonormal tangent vectors for any choice of $\varphi$, (93) can be used with arbitrary $\varphi$. The basis $P_{s}, P_{\vartheta}, P_{\varphi}, P_{\alpha}$ of the KS momentum space can be supplemented by position spinors $U_{j}=P_{j}^{\dagger}$ to obtain the basis of a canonical coordinate system in spinor space. This basis set can then be used to investigate the stability properties of closed orbits.

As a particular application, consider the stability determinant

$$
M^{\prime}=\operatorname{det} \frac{\partial\left(p_{\vartheta_{f}}, p_{\varphi_{f}}\right)}{\partial\left(\vartheta_{i}, \varphi_{i}\right)}
$$

occurring in the closed-orbit theory description of atoms in crossed-fields 12131415$]$. It encodes the stability of a closed orbit starting from the nucleus in the direction characterized by the angles $\vartheta_{i}, \varphi_{i}$ and returning from the direction $\vartheta_{f}, \varphi_{f}$. As it stands, (94) is not well suited to practical computations because it suffers from the singularities of the spherical coordinate chart: At the poles, neither the angle $\varphi$ nor the angular momenta $p_{\vartheta}$ and $p_{\varphi}$ are well defined, so that close to the poles, the calculation of the determinant becomes numerically unstable. With the help of the spinor basis (93), (94) can be rewritten in a form that is not plagued by any singularities.

For a trajectory returning to the nucleus at time $\tau=0$ with a final KS momentum $P_{f}$, the solution (75) of the Kepler equation of motion takes the form

$$
\begin{aligned}
& U(\tau)=\frac{P_{f}^{\dagger}}{\sqrt{-2 E}} \sin (\sqrt{-2 E} \tau)=-\sqrt{\frac{r}{2}} P_{f}^{\dagger}, \\
& P(\tau)=P_{f} \cos (\sqrt{-2 E} \tau)=\sqrt{1+E r} P_{f},
\end{aligned}
$$

which is valid as soon as the electron is sufficiently close to the nucleus so that the external fields can be neglected. In (95), the normalization condition $U^{\dagger} U=2 r$ and the pseudo-energy conservation (61) were used. 
To discuss the stability properties of an orbit, consider a family of orbits parameterized by an arbitrary parameter $\omega_{1}$. Equations of motion for the derivatives $\partial U / \partial \omega_{1}$ and $\partial P / \partial \omega_{1}$ are then obtained by linearizing the equations of motion for $U$ and $P$ around the unperturbed trajectory. Close to the nucleus the dynamics in governed by the linear equations (62), so that the perturbations satisfy the same equations of motion as the coordinates and momenta. In analogy with [63], they read

$$
\begin{aligned}
& \frac{\partial U}{\partial \omega_{1}}=\frac{M_{1}^{\dagger}}{\sqrt{-2 E}} \sin (\sqrt{-2 E} \tau)+M_{2} \cos (\sqrt{-2 E} \tau), \\
& \frac{\partial P}{\partial \omega_{1}}=M_{1} \cos (\sqrt{-2 E} \tau)-\sqrt{-2 E} M_{2}^{\dagger} \sin (\sqrt{-2 E})
\end{aligned}
$$

with constant even multivectors $M_{1}$ and $M_{2}$. With the help of the normalizations inferred from (95), equation (96) simplifies to

$$
\begin{aligned}
& \frac{\partial U}{\partial \omega_{1}}=-\sqrt{\frac{r}{2}} M_{1}^{\dagger}+\sqrt{1+E r} M_{2}, \\
& \frac{\partial P}{\partial \omega_{1}}=\sqrt{1+E r} M_{1}-\sqrt{2 r} E M_{2}^{\dagger}
\end{aligned}
$$

so that the constants $M_{1}=\partial P_{f} / \partial \omega_{1}$ and $M_{1}=\partial U_{f} / \partial \omega_{1}$ can finally be identified with the values of the derivatives obtained at $r=0$.

According to (68), the angular momentum component in a plane specified by a bivector $B$ is

$$
L_{B}=L * B=\frac{1}{2}\langle B U P\rangle .
$$

The derivative of $L_{B}$ with respect to a parameter $\omega_{1}$ can then be calculated with the help of (97). It is given by

$$
\frac{\partial L_{B}}{\partial \omega_{1}}=\frac{1}{2}\left\langle P_{f} B \frac{\partial U_{f}}{\partial \omega_{1}}\right\rangle .
$$

It does not depend on $r$ because all components of the angular momentum bivector are conserved in the Coulomb region.

For the angular momentum component $p_{\omega_{2}}$ conjugate to an angular coordinate $\omega_{2}$, the relevant bivector is

$$
B=s \frac{\partial s}{\partial \omega_{2}},
$$

so that

$$
\frac{\partial p_{\omega_{2}}}{\partial \omega_{1}}=\frac{1}{2}\left\langle P_{f} s \frac{\partial s}{\partial \omega_{2}} \frac{\partial U_{f}}{\partial \omega_{1}}\right\rangle=\left\langle P_{\omega_{2}} \frac{\partial U_{f}}{\partial \omega_{1}}\right\rangle,
$$

where $P_{\omega_{2}}$ denotes the basis spinor corresponding to $\omega_{2}$ by (84) with the final momentum $P_{f}$ used in place of $P_{0}$.

Consider coordinates $\bar{\vartheta}$ and $\bar{\varphi}$, such that $\partial \boldsymbol{s} / \partial \bar{\vartheta}=\boldsymbol{s}_{\vartheta}$ and $\partial \boldsymbol{s} / \partial \bar{\varphi}=\boldsymbol{s}_{\varphi}$ are unit vectors given by (88). The stability determinant $M^{\prime}$ can then be rewritten as

$$
\operatorname{det} \frac{\partial\left(p_{\vartheta_{f}}, p_{\varphi_{f}}\right)}{\partial\left(\vartheta_{i}, \varphi_{i}\right)}=\operatorname{det}\left(\begin{array}{cc}
\frac{\partial p_{\bar{\vartheta}_{f}}}{\partial \bar{\vartheta}_{i}} & \sin \vartheta_{i} \frac{\partial p_{\bar{\vartheta}_{f}}}{\partial \bar{\varphi}_{i}} \\
\sin \vartheta_{f} \frac{\partial p_{\bar{\varphi}_{f}}}{\partial \bar{\vartheta}_{i}} & \sin \vartheta_{i} \sin \vartheta_{f} \frac{\partial p_{\bar{\varphi}_{f}}}{\partial \bar{\varphi}_{i}}
\end{array}\right)=\sin \vartheta_{i} \sin \vartheta_{f} M
$$


with a $2 \times 2$-determinant

$$
M=\operatorname{det}\left(\begin{array}{cc}
\left\langle P_{\vartheta} \frac{\partial U_{f}}{\partial \bar{\vartheta}_{i}}\right\rangle & \left\langle P_{\vartheta} \frac{\partial U_{f}}{\partial \bar{\varphi}_{i}}\right\rangle \\
\left\langle P_{\varphi} \frac{\partial U_{f}}{\partial \bar{\vartheta}_{i}}\right\rangle & \left\langle P_{\varphi} \frac{\partial U_{f}}{\partial \bar{\varphi}_{i}}\right\rangle
\end{array}\right)
$$

free of any coordinate-induced singularities. It is this form of the stability determinant that was used in [15].

The derivatives $\partial U_{f} / \partial \omega$ needed in (103) can be calculated numerically by integrating the linearized equations of motion

$$
\begin{aligned}
\frac{d}{d \tau} \frac{\partial U}{\partial \omega} & =\left(\frac{\partial U}{\partial \omega} * \partial_{U}\right) \partial_{P} \mathcal{H}+\left(\frac{\partial P}{\partial \omega} * \partial_{P}\right) \partial_{P} \mathcal{H}, \\
\frac{d}{d \tau} \frac{\partial P}{\partial \omega} & =-\left(\frac{\partial U}{\partial \omega} * \partial_{U}\right) \partial_{U} \mathcal{H}-\left(\frac{\partial P}{\partial \omega} * \partial_{P}\right) \partial_{U} \mathcal{H}
\end{aligned}
$$

along the closed orbit with initial conditions

$$
\frac{\partial U}{\partial \omega}(0)=0, \quad \frac{\partial P}{\partial \omega}(0)=P_{\omega} .
$$

\section{Conclusion}

The geometric-algebra formulation of the KS transformation endows the four KS coordinates with a clear geometric interpretation. It was shown in this paper that it also allows for an easy incorporation into a Lagrangian and Hamiltonian formulation of the KS theory. At the same time, the known KS Hamiltonian, that was restricted to homogeneous external fields, was generalized to describe the KS motion in arbitrary static external electromagnetic fields. This result is of interest beyond the realm of atomic physics because it is equally applicable to an arbitrary conservative nonelectromagnetic force.

Closed orbits starting at and returning to the Coulomb centre require a special treatment that takes the singularity of the KS coordinate frame into account. The geometric algebra offers a convenient way to derive closed orbit initial conditions and a basis of the spinor space that separates physically distinct degrees of freedom.

The calculations carried out here amply illustrate the power of the geometric algebra formulation of the KS theory. It can thus be expected to form a convenient starting point for analytic calculations in classical perturbation theory.

\section{Appendix A. Introduction to geometric algebra}

Geometric algebra is an algebraic system designed to represent the geometric properties of Euclidean space in the most comprehensive and systematic way possible. It was pioneered by Hermann Grassmann and William Kingdon Clifford during the nineteenth century. From the 1960's on, David Hestenes, with the aim of providing a universal mathematical framework for theoretical physics, extended the algebraic techniques of Grassmann and Clifford by a differential and integral calculus within the geometric algebra, which he called geometric calculus [16].

This appendix gives only a sketch of geometric algebra as far as it is needed in the present work. A more extensive introduction, with an extension to Minkowski spacetime, is contained in [18, 17. A thorough introduction to the geometric algebra of Euclidean 3-space, with a detailed discussion of applications to classical mechanics, 
can be found in [9]. A detailed presentation of the mathematical properties of the geometric algebra is given in [16].

\section{Appendix A.1. The geometric algebra of Euclidean 3-space}

The orientation of two vectors $\boldsymbol{a}$ and $\boldsymbol{b}$ in space can be characterized by the projection of one vector onto the other, which is described by the scalar product $\boldsymbol{a} \cdot \boldsymbol{b}$, and the plane spanned by $\boldsymbol{a}$ and $\boldsymbol{b}$, which is characterized by the vector product $\boldsymbol{a} \times \boldsymbol{b}$. In the geometric algebra these complementary products $\boldsymbol{a} \cdot \boldsymbol{b}$ and $\boldsymbol{a} \times \boldsymbol{b}$ are unified into a single "geometric" product $\boldsymbol{a} \boldsymbol{b}$. The construction of the geometric product starts by picking a right-handed frame of orthonormal unit vectors $\boldsymbol{\sigma}_{1}, \boldsymbol{\sigma}_{2}$ and $\boldsymbol{\sigma}_{3}$. For them, the existence of an associative, but non-commutative geometric product satisfying

$$
\sigma_{i} \sigma_{j}+\sigma_{j} \sigma_{i}=2 \delta_{i j}
$$

is assumed. In addition, the geometric product is required to obey the distributive law with respect to the usual addition of vectors. It follows from th.1 that $\boldsymbol{\sigma}_{i}^{2}=1$ is a scalar. The reader may notice that the defining relation (A.1) is the same as obeyed by the Pauli spin matrices. Indeed, these matrices generate a matrix representation of the Clifford algebra of Euclidean 3-space. In the present context, however, it is important to retain the interpretation of the $\boldsymbol{\sigma}_{i}$ as ordinary vectors instead of regarding them as matrices. The elements of the Clifford algebra are thus given a geometric interpretation, as is indicated by the name "geometric algebra" introduced by Clifford himself. It turns out that all calculations within geometric algebra can be done without recourse to a matrix representation.

By virtue of the defining relation (A.1), the geometric product of two arbitrary vectors $\boldsymbol{a}=\sum_{i=1}^{3} a_{i} \boldsymbol{\sigma}_{i}$ and $\boldsymbol{b}=\sum_{i=1}^{3} b_{i} \boldsymbol{\sigma}_{i}$ is

$$
\begin{aligned}
\boldsymbol{a} \boldsymbol{b}= & a_{1} b_{1}+a_{2} b_{2}+a_{3} b_{3} \\
& \left(a_{2} b_{3}-a_{3} b_{2}\right) \boldsymbol{\sigma}_{2} \boldsymbol{\sigma}_{3}+\left(a_{3} b_{1}-a_{1} b_{3}\right) \boldsymbol{\sigma}_{3} \boldsymbol{\sigma}_{1}+\left(a_{1} b_{2}-a_{2} b_{1}\right) \boldsymbol{\sigma}_{1} \boldsymbol{\sigma}_{2} .
\end{aligned}
$$

The scalar terms of this equation comprise the scalar product $\boldsymbol{a} \cdot \boldsymbol{b}$. In addition, there are terms containing the product of two orthogonal vectors. These terms are neither scalars nor vectors. They are referred to as bivectors. As their coefficients are the components of the vector cross product $\boldsymbol{a} \times \boldsymbol{b}$, bivectors should be interpreted as describing an oriented area in the same way as a vector describes an oriented line segment. Accordingly, a product of three orthonormal vectors is called a trivector and interpreted as representing an oriented volume element. With the help of the unit trivector

$$
I=\sigma_{1} \sigma_{2} \sigma_{3},
$$

equation (A.2) can be rewritten as

$$
a b=a \cdot b+I a \times b,
$$

which achieves the desired unification of the scalar and vector products. Notice that (A.4) contains a sum of quantities of different types, a scalar and a bivector. This should be regarded as a formal sum combining quantities of different types into a single object with a scalar and a bivector part, in analogy to how a real and an imaginary number are added to yield a complex number. 
As the scalar product is symmetric in its factors whereas the vector product is anti-symmetric, these products can be recovered from the geometric product via

$$
\begin{aligned}
& \boldsymbol{a} \cdot \boldsymbol{b}=\frac{1}{2}(\boldsymbol{a} \boldsymbol{b}+\boldsymbol{b a}), \\
& \boldsymbol{a} \times \boldsymbol{b}=\frac{1}{2 I}(\boldsymbol{a} \boldsymbol{b}-\boldsymbol{b a}) .
\end{aligned}
$$

In particular, parallel vectors commute under the geometric product whereas perpendicular vectors anti-commute, and any vector $\boldsymbol{a}$ satisfies $\boldsymbol{a} \boldsymbol{a}=\boldsymbol{a} \cdot \boldsymbol{a}$.

It is a crucial feature of the geometric algebra that it contains elements of different grades, viz. scalars, vectors, bivectors, and trivectors. A general element can be written as a sum of these pure-grade components and is called a multivector. The pure-grade parts of any multivector can be extracted by means of a grade projector. Let $\langle A\rangle_{k}$ be the grade- $k$ part of the multivector $A$. Due to its particular importance, the scalar projector can be abbreviated as $\langle A\rangle=\langle A\rangle_{0}$, and the scalar product of two multivectors $A$ and $B$ is defined by

$$
A * B=\langle A B\rangle \text {. }
$$

For vectors, this agrees with the scalar dot product. Any two multivectors commute under the scalar product:

$$
A * B=B * A .
$$

In a term containing different kinds of products, the scalar product as well as the vector cross product are understood to take precedence over the geometric product. This convention has already been used in A.4.

A multivector which contains only parts of even grades, i.e., scalars and bivectors, is referred to as an even multivector. The even multivectors form a subalgebra of the full geometric algebra.

Finally, the reversion $A^{\dagger}$ of a multivector $A$ is obtained by interchanging the order of vectors in any geometric product. Thus, bivectors and trivectors change sign under reversion, whereas scalars and vectors remain unchanged. Formally, the reversion can be defined by the properties $\boldsymbol{a}^{\dagger}=\boldsymbol{a}$ for any vector $\boldsymbol{a}$ and

$$
(A B)^{\dagger}=B^{\dagger} A^{\dagger}, \quad(A+B)^{\dagger}=A^{\dagger}+B^{\dagger}
$$

for multivectors $A$ and $B$.

Appendix A.2. Rotations in the geometric algebra

Within the geometric algebra rotations are conveniently represented in the form

$$
\boldsymbol{a} \mapsto \mathrm{R}(\boldsymbol{a})=R \boldsymbol{a} R^{\dagger}
$$

with an even multivector $R$ satisfying the normalization condition

$$
R R^{\dagger}=1 \text {. }
$$

Conversely, any normalized even multivector describes a rotation. An arbitrary even multivector satisfies $\alpha=U U^{\dagger} \geq 0$, so that $U=\sqrt{\alpha} R$ is a multiple of a rotor $R$. Therefore,

$$
U \boldsymbol{a} U^{\dagger}=\alpha R \boldsymbol{a} R^{\dagger}
$$

and $U$ describes a rotation-dilatation of 3-space. In particular,

$$
U \boldsymbol{a} U^{\dagger}=\left\langle U \boldsymbol{a} U^{\dagger}\right\rangle_{1}
$$


is a vector for any even multivector $U$ and any vector $\boldsymbol{a}$.

A rotation can be characterized by specifying two vectors $\boldsymbol{a}$ and $\boldsymbol{b}$ so that $\boldsymbol{a}$ is mapped to $\boldsymbol{b}$ by a rotation in the plane $\langle\boldsymbol{a} \boldsymbol{b}\rangle_{2}$ spanned by $\boldsymbol{a}$ and $\boldsymbol{b}$. The rotor $R$ describing this rotation is

$$
R=\frac{1+\boldsymbol{b} \boldsymbol{a}}{|\boldsymbol{a}+\boldsymbol{b}|}=\frac{1+\boldsymbol{b} \boldsymbol{a}}{\sqrt{2(1+\boldsymbol{a} \cdot \boldsymbol{b})}} .
$$

If, alternatively, a rotation is characterized by its rotation axis, given by a unit vector $\boldsymbol{n}$, and a rotation angle $\varphi$, the pertinent rotor reads

$$
R=\mathrm{e}^{-I \boldsymbol{n} \varphi / 2},
$$

where the exponential function of an arbitrary multivector is defined by the familiar power series

$$
\mathrm{e}^{A}=\sum_{n=0}^{\infty} \frac{A^{n}}{n !} .
$$

It satisfies the "power law" relation

$$
\mathrm{e}^{A+B}=\mathrm{e}^{A} \mathrm{e}^{B}
$$

if $A B=B A$ and

$$
\begin{array}{ll}
\mathrm{e}^{A} B=B \mathrm{e}^{A} & \text { if } A B=B A, \\
\mathrm{e}^{A} B=B \mathrm{e}^{-A} & \text { if } A B=-B A .
\end{array}
$$

\section{Appendix A.3. The multivector derivative}

The formalism of the multivector derivative provides a differential calculus for arbitrary multivector functions. Let $F(X)$ be a smooth multivector-valued function of the multivector argument $X$. Neither the grades contained in $X$ nor in $F$ are specified. The directional derivative in the direction of a fixed multivector $A$ by

$$
A * \partial_{X} F(X)=\left.\frac{d F\left(X+\tau P_{X}(A)\right)}{d \tau}\right|_{\tau=0},
$$

where $P_{X}(A)$ projects $A$ onto the grades contained in $X$. A.19) agrees with the familiar definition of the directional derivative.

Let $e_{J}, J=1, \ldots, 8$ be a basis of the geometric algebra and $e^{J}$ its dual basis, i.e., $e_{J} * e^{K}=\delta_{J}^{K}$. The multivector derivative is then defined to be

$$
\partial_{X}=\sum_{J} e^{J} e_{J} * \partial_{X} .
$$

It inherits the algebraic properties of its argument $X$. In particular, $\partial_{X}$ contains the same grades as $X$. Notice that the scalar product $A * \partial_{X}$ is indeed the directional derivative in the direction $A$, justifying the notation introduced in A.19). For a vector argument $\boldsymbol{x}$, the multivector derivative $\partial_{\boldsymbol{x}}$ reduces to the vector derivative, which is analogous to the familiar nabla operator.

Both the directional derivative and the multivector derivative are linear operators and satisfy Leibniz' rule

$$
\begin{aligned}
& A * \partial_{X}(F(X) G(X))=\left(A * \partial_{X} F(X)\right) G(X)+F(X)\left(A * \partial_{X} G(X)\right), \\
& \partial_{X}(F(X) G(X))=\stackrel{*}{\partial}_{X} \stackrel{*}{F}(X) G(X)+\stackrel{*}{\partial}_{X} F(X) \stackrel{*}{G}(X) .
\end{aligned}
$$


In A.22, the overstars indicate the functions to be differentiated. Notice that the second term in A.22 is in general different from $F(X)\left(\partial_{X} G(X)\right)$, because due to its multivector properties the multivector derivative does not commute with $F$ even if $F(X)$ is not differentiated. The directional derivative, on the contrary, is a scalar differential operator that commutes with any multivector that is not to be differentiated. For this reason it is often convenient to write the multivector derivative as

$$
\partial_{X}=\partial_{A} A * \partial_{X}
$$

This form decomposes $\partial_{X}$ into a multivector $\partial_{A}$ and a scalar differential operator $A * \partial_{X}$, which can be moved freely among multivectors.

In addition, the directional derivative satisfies the chain rule

$$
A * \partial_{X} F(G(X))=\left(A * \partial_{X} G(X)\right) * \partial_{G} F(G)
$$

which is useful in many calculations.

A fundamental result concerning the multivector derivative is

$$
\partial_{X}\langle X A\rangle=\partial_{X}\langle A X\rangle=P_{X}(A)
$$

for any multivector $A$. As a consequence,

$$
\partial_{X}\left\langle X^{\dagger} A\right\rangle=\partial_{X}\left\langle A X^{\dagger}\right\rangle=P_{X}\left(A^{\dagger}\right)
$$

\section{References}

[1] Euler L 1765 Novi Comm. Acad. Sci. Petrop. 11144

[2] Levi-Cività T 1956 Opere mathematiche $\mathbf{2}$

[3] Kustaanheimo P 1964 Annales Universitatis Turkuensis. Ser. AI. 73

[4] Kustaanheimo P and Stiefel E 1965 J. Reine Angew. Mathematik 218204

[5] Stiefel E and Scheifele G 1971 Linear and Regular Celestial Mechanics (Springer)

[6] Gourlay M J, Uzer T and Farrelly D 1993 Phys. Rev. A 47 3113, erratum 482508

[7] von Milczewski J and Uzer T 1997 Phys. Rev. E 556540

[8] Sadovskií D A and Zhilinskií B I 1998 Phys. Rev. A 572867

[9] Hestenes D 1990 New Foundations for Classical Mechanics (Kluwer Academic Publishers)

[10] Du M L and Delos J B 1988 Phys. Rev. A 381896 and 1913

[11] Bogomolny E B 1989 Sov. Phys. JETP 69275

[12] Ellerbrock T 1991 Der Zusammenhang zwischen den klassischen Trajektorien und dem Photoabsorptionsspektrum des hochangeregten Wasserstoffatoms in gekreuzten elektrischen und magnetischen Feldern Master's thesis Universität Bielefeld, Germany

[13] Main J 1991 Das hochangeregte Wasserstoffatom im Magnetfeld und in gekreuzten magnetischen und elektrischen Feldern Ph.D. thesis Universität Bielefeld, Germany

[14] Bartsch T 2002 The hydrogen atom in an electric field and in crossed electric and magnetic fields: Closed-orbit theory and semiclassical quantization. (Göttingen, Germany: Cuvillier)

[15] Bartsch T, Main J and Wunner G Semiclassical quantization of the hydrogen atom in crossed electric and magnetic fields phys. Rev. A, submitted. LANL preprint nlin.CD/0212017

[16] Hestenes D and Sobczyk G 1984 Clifford Algebra to Geometric Calculus (D. Reidel Publishing Company)

[17] Lasenby A N, Doran C J L and Gull S F 1993 Found. Phys. 231295

[18] Gull S F, Lasenby A N and Doran C J L 1993 Found. Phys. 231175

[19] Dirac P A M 1933 Proc. Camb. Phil. Soc. 29389

[20] Dirac P A M 1950 Canad. J. Math. 2129 